\title{
Observation of a Cole-Davidson type complex conductivity in the limit of very low carrier densities in doped silicon
}

\author{
Tae-In Jeon and D. Grischkowsky \\ School of Electrical and Computer Engineering and Center for Laser and Photonics Research, \\ Oklahoma State University, Stillwater, Oklahoma 74078
}

(Received 22 January 1998; accepted for publication 2 March 1998)

\begin{abstract}
Using $\mathrm{THz}$ time-domain spectroscopy to measure the complex conductivity of doped silicon from low frequencies to frequencies higher than the $\mathrm{THz}$ plasma frequency and the carrier damping rate, we were able to show in the limit of extremely low carrier densities $N<10^{13} / \mathrm{cm}^{3}$, that the ColeDavidson $(\mathrm{C}-\mathrm{D})$ type complex conductivity accurately describes the conductivity of doped silicon. In the low $\mathrm{N}$ limit the $\mathrm{C}-\mathrm{D}$ parameter $\beta$ converges to 0.83 for $n$-type and 0.70 for $p$-type silicon. In addition, we have observed a new absorption line at $1.9 \mathrm{THz}$ from an unidentified defect in some of our Czochralski, single-crystal, low-N silicon samples. (O) 1998 American Institute of Physics. [S0003-6951(98)01518-6]
\end{abstract}

Although the complex conductivity of doped semiconductors has been a topic of theoretical studies for several decades, complete experimental characterizations from low frequencies to beyond several $\mathrm{THz}$ have only started to be performed, ${ }^{1-3}$ due to the difficulty of reaching this frequency region with conventional sources. The relatively new technique of Thz time-domain spectroscopy (THz-TDS) has enabled such experimental studies, ${ }^{1-3}$ which have begun to test the theories of conductivity. The most accurate and recent measurements ${ }^{3}$ showed that the complex conductivity of doped silicon did not fit any standard theory, but was in good agreement with an alternative approach based on a ColeDavidson (C-D) type distribution. ${ }^{4}$ This was the first application of a C-D type distribution to fit the complex conductivity of well-ordered crystalline semiconductors; it extended the demonstrated high frequency limit of this type of distribution by more than three orders of magnitude.

Previously the C-D distribution has been associated with the Debye theory ${ }^{4}$ of dielectric insulators. For relatively low frequencies, the complex dielectric constants of disordered materials such as molecular liquids, ${ }^{4}$ polymers, ${ }^{5}$ and more recently ionic glasses, ${ }^{6.7}$ show better agreement with a C-D distribution, than Debye theory. The Debye theory is closely related to Drude theory, ${ }^{8}$ the simplest theory of electrical conduction. In the frequency domain the mathematical representations of Debye and Drude theories are identical. The C-D distribution corresponds to Debye theory with a fractional exponent $\beta$, limited to values between 0 and 1 , and reduces to Debye theory for $\beta=1$. In the most recent $\mathrm{THz}$ TDS work on silicon, ${ }^{3}$ the frequency-dependent complex conductivity of Drude theory was similarly modified to include the $\mathrm{C}$-D fractional exponent $\beta$, and thereby, achieved an exceptional fit to the measurements. It was observed that $\beta$ approached unity (Drude theory) as the number density $N$ of carriers was increased for both $n$ - and $p$-type silicon. However, the measurements could not establish whether $\beta$ approached limiting values as $N$ was decreased. These limits are important to understand the nature of conductivity and to test whether fractal conductivity can occur in crystalline material. The C-D distribution can arise from conduction on materials with fractal geometries or fractal time processes where a temporal cutoff is included which limits the fractal conduction to well defined time scales. For example, Niklasson $^{7,9}$ has stated that a C-D type distribution can be obtained from a fractal conduction process with a cutoff and relating this to percolation theory would predict $\beta=0.72$.

Here we report THz-TDS measurements which show that in the limit of low carrier densities the complex conductivity continues to be in excellent agreement with the C-D type distribution and the C-D parameter $\beta$ converges to 0.83 for $n$-type and to 0.70 for $p$-type silicon. The range of the measurements extended from 0.08 to $3.5 \mathrm{THz}$ and included the plasma frequency and the carrier damping rate. The custom fabricated samples are the highest resistivity, lowest carrier density, samples of silicon ever completely characterized. Their measurement called for a different experimental procedure and set a new standard of sensitivity for $\mathrm{THz}$ TDS. The high sensitivity has enabled us to observe a new absorption line at $1.9 \mathrm{THz}$ from an unidentified defect in some of our Czochralski $(\mathrm{Cz})$, single-crystal, low-N silicon samples.

The THz-TDS measurements were performed by optoelectronically measuring the amplitude of freely propagating subpsec $\mathrm{THz}$ electromagnetic pulses transmitted through the silicon sample under investigation. ${ }^{1-3,10}$ These transmitted pulses were then compared to the measured $\mathrm{THz}$ pulses with the sample replaced by an identical cylinder of high resistivity, greater than $5 \mathrm{k} \Omega \mathrm{cm}$, float zone (FZ) Si with a residual carrier density of less than $5 \times 10^{11} / \mathrm{cm}^{3}$, less than $1 / 10$ that of our lowest $\mathrm{N}$ sample. Using this procedure the power transfer function of the THz-TDS system was unchanged by the addition of large samples with high dielectric constants, and the measurements were directly compared to highresistivity $\mathrm{FZ}-\mathrm{Si}$. This procedure allowed the measurement of the extremely small absorption on the high frequency wing of the sample response. The $\mathrm{THz}$ system was in an enclosure maintained at a relative humidity of less than $2 \%$ to mitigate the effects of water vapor. Analysis of the respective complex numerical Fourier transforms determines the frequency dependent absorption and index of refraction. ${ }^{10}$ 
The three $n$-type (phosphorus) cylindrical samples were: $30 \Omega \mathrm{cm}$ ( $4 \mathrm{~cm}$ long), $50 \Omega \mathrm{cm}(2 \mathrm{~cm}$ long), $5.1 \mathrm{~cm}$ diam. $\mathrm{Cz}-\mathrm{Si}$, and $320 \Omega \mathrm{cm}(15 \mathrm{~cm}$ long $) 7.6 \mathrm{~cm}$ diam., neutrondoped FZ-Si. The two $p$-type (boron) samples were: $44 \Omega \mathrm{cm}(4 \mathrm{~cm}$ long $)$ and $55 \Omega \mathrm{cm}(2 \mathrm{~cm}$ long $) 5.1 \mathrm{~cm}$ diam. $\mathrm{Cz}-\mathrm{Si}$. The $\mathrm{Cz}-\mathrm{Si}$ samples were obtained from Kamis Inc.; the $320 \Omega \mathrm{cm}$, FZ-Si sample was from UniSil Corp.; the high-resistivity FZ-Si reference cylinders were from UniSil Corp. and TopSil Corp. All the Si samples and reference cylinders had $\langle 100\rangle$ orientation.

The frequency-dependent complex dielectric constant $\epsilon$ is equal to the square of the complex index of refraction $n$ $=n_{r}+i k$. The imaginary index $k$ is determined by measuring the power absorption coefficient $\alpha=k 4 \pi / \lambda_{0}$, where $\lambda_{0}$ is the free-space wavelength. The dielectric response for doped silicon is described by the following general relationship, $\epsilon=\epsilon_{\mathrm{Si}}+i \sigma /\left(\omega \epsilon_{0}\right)$, where $\epsilon_{\mathrm{Si}}$ is the dielectric constant of intrinsic silicon, $\sigma$ is the complex conductivity, and $\epsilon_{0}$ is the free-space permittivity. The index of refraction $n_{r}$ $=3.417$ and power absorption coefficient $\alpha<0.05 \mathrm{~cm}^{-1}$ have been measured ${ }^{10}$ for undoped, high-resistivity FZ-Si over the frequency range considered here. For the C-D distribution the conductivity is given by ${ }^{3}$

$$
\sigma=\epsilon_{0} \omega_{l}^{2} \tau /(1-i \omega \tau)^{\beta}
$$

where the plasma frequency $\omega_{p}$ is given by $\omega_{n}^{2}=N e^{2} /\left(\epsilon_{0} m^{*}\right) ; m^{*}=0.26 m_{0}$ for the effective electron mass and $m^{*}=0.37 m_{0}$ for the effective hole mass; $m_{0}$ is the free-electron mass. For Drude theory $\beta=1$. The dc conductivity is given by $\sigma_{\mathrm{dc}}=e \mu N$, with the mobility $\mu=e /\left(\Gamma m^{*}\right)$. The carrier damping rate is $\Gamma=1 / \tau$, where $\tau$ is the carrier collision time.

Our measured power absorption coefficient and index of refraction for the $320 \Omega \mathrm{cm} n$-type sample are shown in Fig. 1(a). Even though the absorption and the changes in the index are extremely low, the data have an excellent signalto-noise ratio due to the long $15 \mathrm{~cm}$ sample length and due to the use of an identical reference cylinder of high-resistivity FZ-Si. As can be seen the agreement with the C-D distribution is excellent over the entire frequency range for both curves. For comparison Drude theory is shown as the dashed line. Although Drude theory fits well at frequencies up to $\Gamma / 2 \pi=0.41 \mathrm{THz}$, at higher frequencies Drude theory falls off much faster than the measured absorption. At the highest frequency the measured absorption is more than twice that predicted by Drude theory. Although the two results of Fig. I(a) are related by the Kramers-Kronig relationships, they are measured independently. The ability to simultaneously obtain good fits with the same parameters for both the absorption and index of refraction shows the accuracy of THz-TDS to measure the complex transmitted spectrum.

Given these measurements of the absorption and index of refraction, the general relationship for $\epsilon$ determines the real $\sigma_{r}$ and the imaginary $\sigma_{i}$ parts of the conductivity shown in Fig. 1(b). The experimental results for $\sigma_{r}$ are only well fit by the C-D distribution, whose fitting line almost vanishes into the presented data. The calculated C-D distribution also fits well to $\sigma_{i}$, which differs significantly with that predicted by Drude.

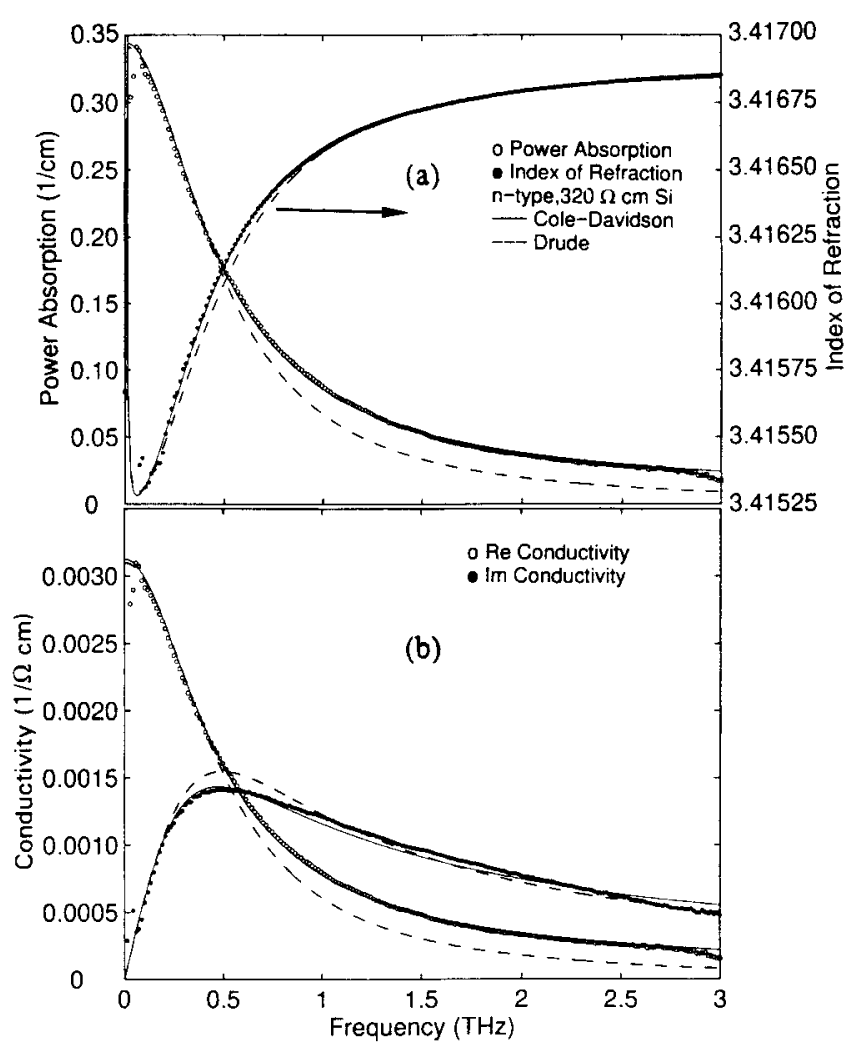

FIG. 1. Measurements for $320 \Omega \mathrm{cm}, n$-type (phosphorus) neutron-doped FZ-Si compared with theory.

The absorption measurements on the four $\mathrm{Cz}-\mathrm{Si}$ samples are shown in Fig. 2. The lower mobility of the $p$-type material can be seen from an inspection of the curves, where the crossovers emphasize their different widths and corresponding different $\Gamma$, inversely proportional to the mobility. Again, with the exception of the newly observed resonance line at $1.9 \mathrm{THz}$, we obtain good agreement with the C-D distribution (solid lines) for all the samples. In Fig. 2(b), it is seen that the resonance feature has vanished for the $n$-type sample, but is still observed for the $p$ type. The differences between the measured absorption and the corresponding best fit C-D distributions for the measurements in Figs. 2(a) and 2(b) are presented in Fig. 2(c) with a $40 \times$ expanded vertical scale. The $1.9 \mathrm{THz}$ resonance and the $0.4 \mathrm{THz}$ FWHM linewidth is the same for both the $n$ and $p$-type samples that show the weak absorption line. This observation and the fact that the feature is sometimes absent for very similar samples, leads us to the conclusion that it is a growth induced defect, probably involving oxygen due to its high concentration in Cz-Si. ${ }^{1}$ Although the identification of this defect remains a future challenge, THz-TDS is clearly the method of choice for its characterization.

Figure 3 summarizes the above results together with the associated series of measurements of Ref. 3 , by presenting the values of the mobilities, carrier densities and the C-D parameters $\beta$ obtained from the best fits to experiment. The mobilities monotonically increase with decreasing carrier density, thereby confirming that the main scattering impurities are the $n$-or $p$-type dopant. This dependence of the mobility on carrier density is well fit by the empirical Caughey-Thomas curve. ${ }^{12}$ It is important to note that our results for $\mathrm{Cz}-\mathrm{Si}$, neutron-doped $\mathrm{FZ}-\mathrm{Si}$ and the previous 


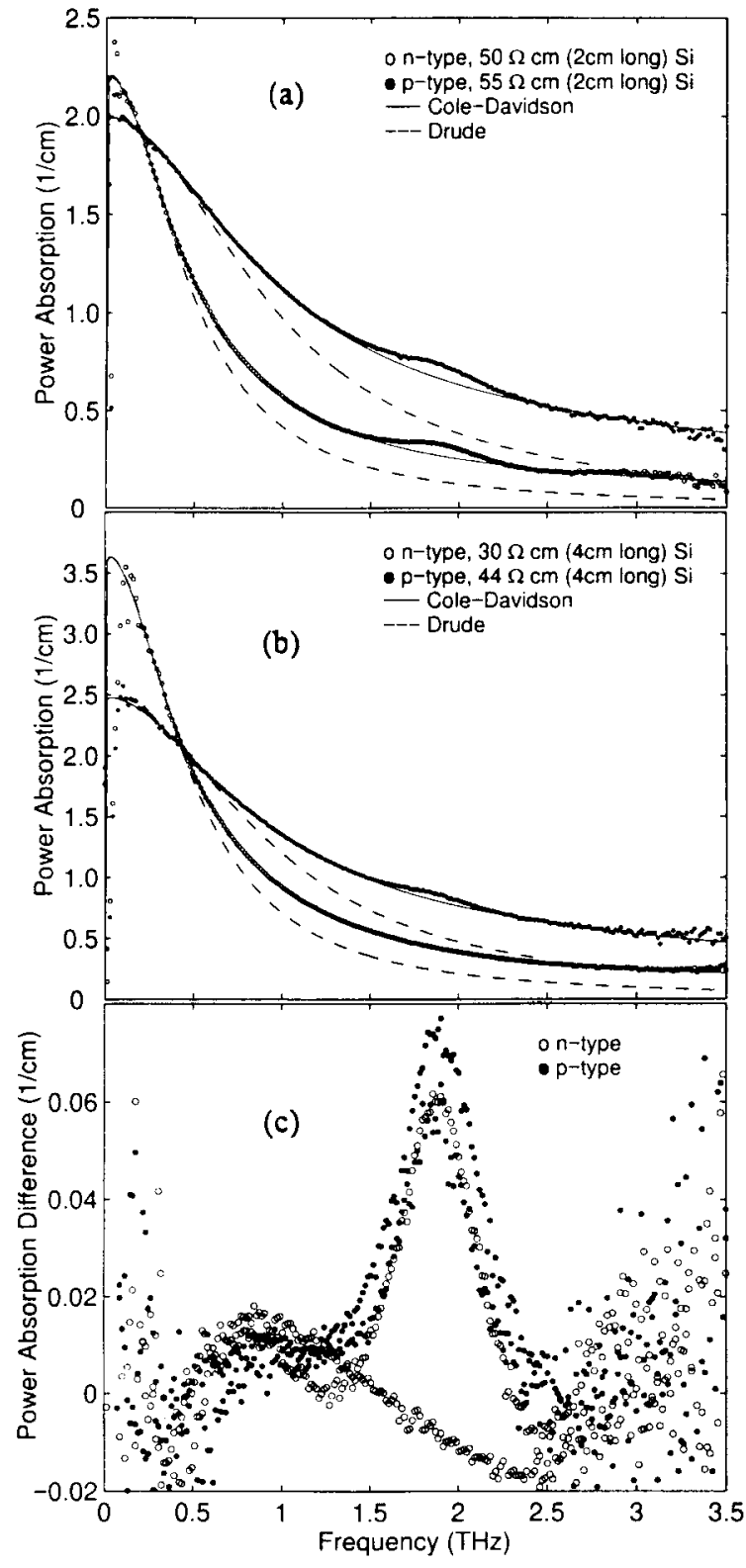

FIG. 2. (a). (b) Measured power absorption for the Cz-Si samples compared with theory. (c) Differences between the measured absorptions and C-D theory: the highest peak is for the $50 \Omega \mathrm{cm}, p$-type sample and the missing peak is for the $30 \Omega \mathrm{cm}, n$-type sample.

work all fit on the same curve. At the lower carrier densities the C-D mobilities are much higher than previous measurements. ${ }^{12}$ At carrier densities above $10^{17} / \mathrm{cm}^{3}$ these differences disappear. For the $n$-type samples with the two highest number densities $\beta=1$, and C-D theory has thereby

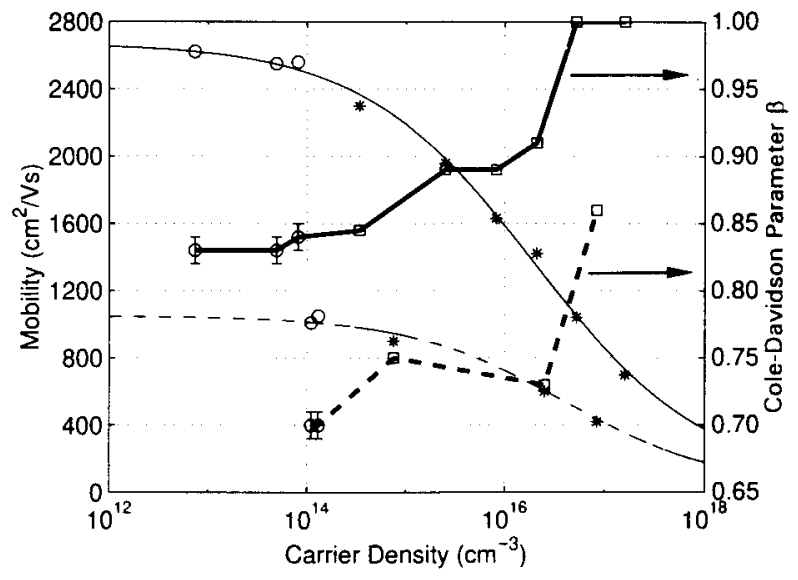

FIG. 3. Values of the mobility and C-D parameter $\beta$ vs carrier number densities obtained from the best C-D theory fits to our measurements (circles) and the measurements of Ref. 3 (asterisks and squares) for the $n$-type (solid lines) and $p$-type (dashed lines) Si samples.

reduced to Drude theory. As the number density is decreased, $\beta$ monotonically decreases and converges to $\beta=0.83 \pm 0.01$ in the limit of very low carrier densities. For the $p$-type samples, $\beta$ shows a more abrupt decrease as the number density is reduced, and then converges to $\beta=0.70$ \pm 0.01 . The estimated uncertainty of \pm 0.01 was determined numerically from our fitting routine. Outside of this range the quality of the C-D fit was severely degraded. The consistency of all the measurements gives confidence in the fundamental nature of the dependence presented in Fig. 3.

The authors acknowledge stimulating and informative discussions with R. A. Cheville and R. W. McGowan. This work was partially supported by the National Science Foundation and the Army Research Office.

'M. van Exter and D. Grischkowsky, Appl. Phys. Lett. 56, 1694 (1990); Phys. Rev. B 41, 12140 (1990).

${ }^{2}$ N. Katzenellenbogen and D. Grischkowsky, Appl. Phys. Lett. 61. 840 (1992).

${ }^{3}$ Tae-In Jeon and D. Grischkowsky, Phys. Rev. Lett. 78, 1106 (1997).

${ }^{4}$ D. W. Davidson and R. H. Cole, J. Chem. Phys. 19, 1484 (1951).

${ }^{5}$ S. Havriliak, Jr. and D. G. Watts, Polymers 27, 1509 (1986).

${ }^{6}$ K. Pathmanathan and J. R. Stevens. J. Appl. Phys. 68, 5128 (1990).

${ }^{7}$ G. A. Niklasson, J. Appl. Phys. 62, RI (1987).

${ }^{8}$ N. W. Asheroft and N. D. Mermin. Solid State Physics (Holt, Rinehart. and Winston. New York, 1976).

${ }^{9}$ G. A. Niklasson, K. Brantervik, and L. Borjesson. J. Non-Cryst. Solids 131, 1096 (1991)

${ }^{10}$ D. Grischkowsky, S. Keiding, M. van Exter, and Ch. Fattinger. I. Opt. Soc. Am. B 7, 2006 (1990).

${ }^{11}$ L. I. Murin, T. Hallberg, V. P. Markevich, and J. L. Lindstrom, Phys. Rev. Lett. 80. 93 (1998).

${ }^{12}$ D. M. Caughey and R. F. Thomas, Proc. IEEE 50, 2192 (1967). 